\title{
EDUCACIÓN E INSERCIÓN SOCIAL: PROBLEMÁTICA ACTUAL DE LA EDUCACIÓN EN ADOLESCENTES Y JÓVENES BAJO MEDIDAS DE INTERNAMIENTO EN EL SALVADOR
}

EdgarVentura

CENICSH

edgar.ventura@mined.gob.sv

Recepción: 4 de enero de 2016

Aceptación: 18 de enero de 2016 


\section{RESUMEN}

El presente trabajo constituye un diagnóstico de la situación actual referente a la atención educativa que reciben los menores de edad en los Centros Educativos adscritos a los Centros de Inserción Social (CIS) en El Salvador. El trabajo sigue una metodología de análisis y reflexión de la situación global de la educación en lo referente a acceso y permanencia en los primeros años de escolaridad; reseña las principales características del proceso educativo en los CIS que ponen de manifiesto las virtudes y limitantes del mismo para conseguir la efectiva inserción social de los jóvenes sujetos de cometimiento de delitos identificando, entre otros aspectos, dos limitantes decisivas que son la falta de especialización docente en educación en contextos de encierro y la inexistencia de lineamientos específicos que orienten el tratamiento educativo. Finalmente, concluye con recomendaciones básicas orientadas al mejoramiento de los proceso socio educativos que tienen lugar en los CIS.

\section{Palabras clave}

Inserción social - Deserción escolar Oportunidades educacionales - Educación acelerada

\section{ABSTRACT}

This work is a diagnosis of the current concerning educational care given to minors in those attached to the Social Integration Centers (CIS, for its acron$\mathrm{ym}$ in Spanish) in El Salvador Educational Centers situation. The work follows a methodology of analysis and reflection of the overall situation of education in terms of access and permanence in the early years of schooling; outlines the main features of the educational process in the CIS that reveal the strengths and limitations of the same to achieve effective social integration of young subjects commission of crimes by identifying, among other aspects, two decisive limitations are the lack of teaching specialization in education, prison inmates and the lack of specific guidelines to guide the educational treatment. Finally, it concludes with key recommendations aimed at improving educational partner taking place in the CIS process.

\section{Keywords}

Social Integration - School Dropout Educational Opportunities - Accelerated Education 


\section{EDUCACIÓN E INSERCIÓN SOCIAL: PROBLEMÁTICA ACTUAL DE LA EDUCACIÓN EN ADOLESCENTES Y JÓVENES BAJO MEDIDAS DE INTERNAMIENTO EN EL SALVADOR}

EdgarVentura

CENICSH

edgar.ventura@mined.gob.sv

\section{Introducción}

El presente trabajo busca facilitar la comprensión de aquellos contextos en los que tiene lugar la educación que reciben adolescentes y jóvenes que cumplen medidas de internamiento a causa de que han sido encontrados sujetos de responsabilidad penal. El punto de partida del trabajo es que la educación envuelve amplias potenciales que contribuyen sustancialmente a los procesos de inserción social que atraviesan aquellos que se han desviado de los marcos normativos socialmente establecidos.

En los contextos educativos, la situación de los jóvenes sujetos de responsabilidad penal se presenta como un problema social cuyas causantes deben verse en forma múltiple, en sus diferentes dimensiones y también como un problema grave que actualmente atraviesa el país debido al modo en que se han ido complejizando estructuras delincuenciales agrupadas bajo la forma de pandillas. El problema de estos jóvenes sujetos de responsabilidad y el asunto del tratamiento que reciben aquellos que cumplen medidas de 
internamiento en los CIS no puede verse desligado de la grave situación de pandillas que atraviesa nuestro país.

Múltiples son las causantes sociales de la violencia que experimenta el país, sin embargo, analizo un aspecto esencial en toda sociedad, motor del progreso social e individual: la educación. A través de ésta es viable la autorrealización de los individuos, mientras su ausencia planteará situaciones adversas y conflictivas. El breve análisis propuesto muestra solamente el aspecto más general del problema relacionado al acceso y permanencia de niños, adolescentes y jóvenes en el sistema educativo. El análisis de las cifras refleja una situación preocupante, pues amplios sectores sociales que quedan fuera del sistema educativo no gozan de los beneficios de corto y largo plazo que puede brindar la educación. Éste es un aspecto que no puede verse desde el así llamado enfoque de la prevención, sino que se trata de darle a la población aquellas condiciones mediante las cuales pueda realizarse como persona y ser funcional a una sociedad que atraviesa grandes desequilibrios.

La parte diagnóstica y de mayor peso en el trabajo se concentra en mostrar las condiciones en que tiene lugar el proceso socio educativo de los jóvenes en los CIS. Se trata de analizar la idoneidad de aquellas condiciones e igualmente mostrar lo complejo de la labor docente bajo las mismas. En ambos aspectos se señalan logros, pero también limitantes que aún es preciso mejorar. Esta parte del trabajo tiene un carácter descriptivo que permite al final señalar una serie de medidas que deben tomarse en lo inmediato a fin de mejorar los procesos educativos en estos contextos; vale mencionar que se ha logrado elaborar gracias al trabajo de campo realizado en las visitas a los CIS, asimismo a las entrevistas que me proporcionaron directores y maestros de los CE adscritos a los CIS, y también bajo el cotejo de las estadísticas en las bases de información del Ministerio de Educación. ${ }^{1}$

\footnotetext{
${ }^{1}$ No omito expresar mi agradecimiento al Subdirector de Inserción Social del ISNA, Ricardo Vladimir Montoya, quien me facilitó el ingreso a los Centros de Inserción Social y poder realizar las entrevistas la personal docente de los Centros Educativos, a quienes igualmente reconozco su valiosa colaboración.
} 


\section{Contexto actual de violencia: el problema de seguridad en cifras}

En el contexto actual de nuestro país, en el que el tema de la seguridad ocupa los primeros lugares dentro de las preocupaciones de la población, se vuelve necesario definir una estrategia por partida doble que apueste seriamente a superar esta dificultosa situación. En mi opinión, esta estrategia debe girar fundamentalmente en torno a dos ejes: recuperar a aquellas personas que se han desviado de las normas socialmente establecidas a una vida normal; y de otra parte, establecer las líneas generales de un proyecto de desarrollo social de largo plazo que permita reducir al mínimo las causas sociales de la violencia. En el análisis que realizaré de la situación macro apuntaré algunas ideas que pueden contribuir a la realización de ambos objetivos que sin lugar a dudas son complejos, sobre todo en una realidad que arroja cifras como las que someramente describiré a continuación.

Esto que actualmente llamamos el problema de seguridad se ha reflejado en el alto índice de homicidios, robos, extorsiones y otros flagelos, cuyos principales responsables son en buena medida estructuras delincuenciales que han ido aumentando su capacidad logística y organizacional, las cuales en sus diferentes vertientes y divisiones se han agrupado bajo la forma de pandillas.

Para ilustrar esta situación de violencia que atraviesa el país basta con mostrar algunas cifras que vuelven la situación verdaderamente preocupante. Producto del cometimiento de actos delictivos que en su mayoría se concentran en el homicidio, robo y extorsión, que constituyen el 58.68\% de los delitos de mayor incidencia en la población que se encuentra recluida en las penitenciarías, el año 2013 cerró con 26,848 personas privadas de libertad en los Centros Penitenciarios. Esto cifra supone de cada 100,000 habitantes aproximadamente 400 se hallan recluidos en alguna cárcel. ${ }^{2}$ Los

\footnotetext{
${ }^{2}$ Esta cifra es incluso mayor que la de países de mayor de la región con mayor extensión territorial y con una mayor cantidad de población como Brasil y México.
} 
rangos de edad de las personas recluidas en estos Centros corresponde en su mayoría a población joven: el 70.62\% de los privados tiene entre 18 y 35 años. Una de las características de esta población es su bajo nivel educativo: el 72.31\% no ha superado los estudios básicos y el 7.79\% no sabe leer ni escribir, o sea, estamos hablando que más del $80 \%$ de esta población tiene un nivel por debajo de lo normal de escolaridad. ${ }^{3}$ Este dato nos servirá más adelante para mostrar la relación causal entre delincuencia y ausencia de oportunidades de acceso a la educación.

Que todos estos hechos delictivos, principalmente los homicidios, están relacionados a las organizaciones pandilleriles se comprueba en el hecho de que entre 2010 y 2013 el porcentaje de aquellos vinculados a las pandillas ha oscilado entre un $15.6 \%$ a un $26.3 \%$. En esta misma línea es preocupante que la cantidad de personas con filiación pandilleril se contabilizaba para 2013 en aproximadamente 34,000 personas, a lo cual debe sumarse la estructura de colaboradores, familiares u otros que contribuyen a los fines de estas organizaciones. En esta misma línea, es de mencionar que para 2014, 9,357 pandilleros se encontraban encarcelados en algún Centro Penitenciario, esto es, aproximadamente un 35\% de la población reclusa. ${ }^{4}$

En relación a los homicidios, el año 2013 cerró con un total de 2,499 personas asesinadas, cantidad que se vio reducida en relación a los años anteriores debido a la mediática tregua entre pandillas, pues para el caso de 2011 fueron 4,371, personas asesinadas, mientras que en 2012 fueron 2,594. En suma, en estos tres años tenemos que 9,464 personas fueron asesinadas. Es importante mencionar que los mayores porcentajes de rangos de edad de las personas asesinadas son similares al de la población recluida, es decir, que se trata de población joven. Por ejemplo, en el año 2013 el 65.9\% de

\footnotetext{
${ }^{3}$ Información disponible en: http://publica.gobiernoabierto.gob.sv/institutions/direcciongeneral-de-centros-penales/information_standards/estadisticas. Última consulta: 22-01-15

${ }^{4}$ Datos retomados del siguiente artículo:Amaya, Luis Enrique y Martínez, Juan José. "Sureños en El Salvador: un acercamiento antropológico a las pandillas de deportados" en Realidad y Reflexión, Enero-Junio, N 39, 2014, 19, 23. Este porcentaje está calculado con el dato de cierre del año 2013 de la población recluida.
} 
los homicidios se cometieron en contra de población oscilaba entre los $15 \mathrm{y}$ los 35 años. Nuevamente, este dato evidencia la pérdida que sufre el país de aquella población que debería estarse formando y capacitando para aportar al desarrollo social, pero lastimosamente la realidad es otra. ${ }^{5}$

\section{Los esfuerzos interinstitucionales en pro de la inser- ción social}

El concepto de inserción social que manejan las autoridades de las instituciones responsables de darle un tratamiento al menor de edad que cumple medidas de internamiento se encuentra justificado a partir de las normas internacionales ${ }^{6} \mathrm{y}$ de la leyes del país vinculadas al tratamiento de adolescentes sujetos a responsabilidad penal juvenil que por motivo de una conducta anti social han incurrido en delitos o faltas a la ley. Para el caso deben mencionarse dos instrumentos jurídicos fundamentales relacionados a esta problemática: La Ley Penal Juvenil, que establece los derechos y deberes de los adolescentes que han cometido una falta, y la Ley de Protección Integral de la Niñez y la Adolescencia, donde se establecen las competencias de instituciones responsables en el tratamiento al menor como lo es el ISNA y la Autoridad Judicial.

La inserción social es vista como un proceso complejo que comprende múltiples dimensiones (social, educativa, familiar) y que, por consiguiente, requiere un conjunto de medidas interinstitucionales "encaminadas a potenciar las estrategias de intervención” en los adolescentes sujetos a

\footnotetext{
${ }^{5}$ Instituto de Medicina legal, “Total de homicidios año 2013, según base de datos del Instituto de Medicina Legal, cotejada y consensuada con F.G.R. y la P.N.C." www.gobiernoabierto. gob.sv. Ultima consulta: 30-01-15

${ }^{6}$ Para el caso deben mencionarse: la Convención de los Derechos del Niño (1989), las Reglas Mínimas de las Naciones Unidas para la administración de la justicia de menores (Reglas de Beijing, 1985) y las Directrices de las Naciones Unidas para la prevención de la delincuencia juvenil (Directrices de Riad, 1990)
} 
responsabilidad penal juvenil. ${ }^{7}$ Se conciben tres programas operativos para el tratamiento a esta población adolescente: un programa dirigido medidas administrativas de resguardo ${ }^{8}$ otro dirigido a la atención en medidas de medio abierto 9 y, finalmente, un programa de atención bajo medidas de internamiento. ${ }^{10}$ La elaboración de estos documentos institucionales que establecen el protocolo a seguir para el tratamiento de adolescentes sujetos de responsabilidad penal juvenil refleja un importante avance para el país en términos de normas de justicia para menores de edad. Esto previene, entre otras cosas, actos arbitrarios en el proceso por el que los adolescentes sujetos de responsabilidad penal juvenil atraviesa desde su captura hasta su proceso de condena por parte de los jueces de menores.

Por otra parte, la finalidad del proceso de inserción social consiste en que los adolescentes experimenten cambios sustanciales en su comportamiento, pero también adopten una nueva perspectiva frente a la vida y puedan definir un proyecto que les permita avanzar hacia metas de corto y largo plazo. Esto puede lograrse mediante la incorporación del adolescente a un proceso socio-educativo que se da bajo el acompañamiento de las instituciones pertinentes y sobre todo de la familia. El modo en que va teniendo lugar este proceso también es complejo, lo cual detallaré cuando desarrolle la experiencia concreta de los adolescentes que cumplen medidas de internamiento en los CIS que están insertos en un proceso socio educativo.

Las principales instituciones involucradas en este proceso de aquellos que cumplen medidas de internamiento son: el Ministerio de Justicia y Seguridad Pública (MJSP), el Instituto Salvadoreño para el Desarrollo Integral de la Niñez y la Adolescencia (ISNA) y el Ministerio de Educación

\footnotetext{
${ }^{7}$ ISNA. Programa marco para la atención integral de adolescentes sujetos a responsabilidad penal juvenil. San Salvador, Mayo de 2013, 7-31, especialmente 7-8

${ }^{8}$ ISNA. Programa de atención en la medida administrativa de resguardo.

${ }^{9}$ ISNA. Programa de atención en las medidas en medio abierto.

${ }^{10}$ ISNA. Programa de atención en la medida de internamiento.
} 
(MINED). ${ }^{11}$ La primera tiene bajo su control los Centros Penales y los Centros Intermedios, cada una administrativamente regida por una Dirección General. ${ }^{12}$ En ambos tipos de Centros funcionan Centros Educativos que por su naturaleza pública se encuentran bajo la jurisdicción del MINED en lo referente al funcionamiento administrativo, asignación presupuestaria y demás elementos del proceso educativo como los programas educativos, entre otros.

Por su lado, el ISNA cumple la función de administrar los así llamados Centros de Inserción Social (CIS), que son los lugares donde se encuentran adscritos los Centros Educativos que brindan oportunidad de cursar la educación básica y media a los jóvenes que cumplen medidas de internamiento. En el curso del trabajo aludiré a cinco Centros Escolares que se ubican en estos últimos. Mientras que en lo relacionado a las penitenciarías, a los cuales me referiré principalmente solo de modo comparativo, he obtenido información de 16 Centros Escolares.

A fin de ampliar mi análisis que permitirá comprender algunas de las causas que motivan a los adolescentes a desviarse de un comportamiento adecuado presento a continuación un análisis de la situación educativa nacional que permitirá esbozar algunas probables explicaciones en torno al asunto.

\footnotetext{
11 Otras instituciones que tienen participación son: el Ministerio de Salud, la Secretaría de Inclusión Social, la Procuraduría General de la República y organizaciones de la sociedad civil (oenegé e iglesias)

${ }^{12}$ La Dirección General de Centros Intermedios, surge según decreto número 20 del Órgano Legislativo, del 15 de junio de 2006, publicado en el Diario Oficial $N^{\circ}$ 126, Tomo 375, año 2006, mediante la reforma realizada a la Ley Penal Juvenil, al Art. 119 Inc. $3^{\circ}$ el cual literalmente establece: "Habrán centros intermedios para el cumplimiento de la medida de internamiento en los casos de personas que hayan cumplido los dieciocho años de edad, que requieran un tratamiento especializado o que su permanencia en el centro implique un perjuicio para los menores de edad". De otra parte se encuentra la Dirección General de Centros Penales.
} 


\section{Situación problemática del estado actual del acceso y permanencia en el sistema educativo}

Cuando analizamos en detalle la situación global de la población reclusa tanto en los CP como en los CIS, puede apreciarse los bajos niveles de escolaridad que esta población posee. Calculando los años de escolaridad de esta población reclusa nos arroja un resultado que no es muy diferente de la situación global del país. En el caso de los CP los años de escolaridad, tomando como base el dato de los matriculados en algún nivel de instrucción, es de 5,8, mientras que en los CIS es de $6,7 .{ }^{13}$ A continuación compararé estos datos con la situación general del país.

Ciertamente, este problema de los bajos niveles de años de escolaridad no concierne solamente a las personas que están en los centros de internamiento, pues según el dato proporcionado por la Encuesta de Hogares de Propósitos Múltiples de 2013 el total de la población salvadoreña posee una escolaridad promedio de 6.6 años de estudio. Esto realmente constituye un problema, pues se esperaría que el promedio sea de nueve años que corresponden a la educación básica, lo cual es el mínimo de educación que todo ciudadano de El Salvador debe tener.

El dato desagregado por zona nos muestra una tendencia a mayores años de escolaridad para la zona urbana (7.7), mientras que en la zona rural la situación es aún más delicada pues apenas y este segmento de la población tiene un promedio de 4.7 años de escolaridad. Éste es un problema de acumulación histórica que tiene diferentes causantes, reflejadas en los bajos niveles de inversión en educación que se traducen en limitada infraestructura educativa, déficit en la cantidad de maestros para tender al total de la población en edad escolar que ingresa al primer año de primaria, entre otros. ${ }^{14}$ En la siguiente gráfica se ilustra la situación de los años de escolaridad según zona urbana y rural:

\footnotetext{
${ }^{13}$ Elaboración propia en base a Censo Escolar 2013 del MINED

${ }^{14}$ Ver mi trabajo: "El acceso a la educación primaria y la reforma educativa salvadoreña (1960-1972)" en Revista de Humanidades y Ciencias Sociales, № 6, Enero-Junio, 2014, 63-90 
GRÁFICA 1 AÑOS DE ESCOLARIDAD A NIVEL NACIONAL DESAGREGADO
POR ZONA

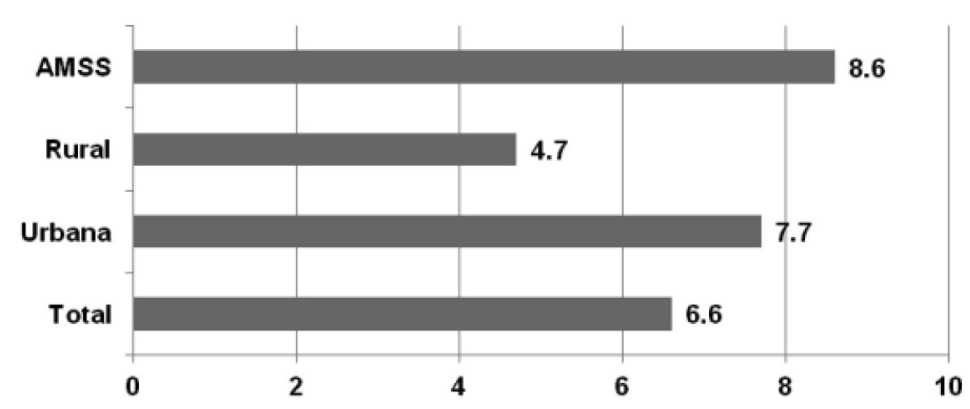

Fuente: Encuesta de Hogares de Propósitos Múltiples, 2013, 7

Las anteriores cifras nos muestran un fragmento de la realidad del país que reflejan las limitaciones que aún persisten en cuanto al acceso a la educación. Las consecuencias sociales que esto conlleva se traducen en buena medida en el problema actual de violencia e inseguridad que predomina en el país. ${ }^{15}$ Por consiguiente, nos plantea analizar una realidad micro, cual es la educación para la población privada de libertad, en un contexto general más amplio y más complejo, pues por una parte el dato que nos muestra los niveles educativos de la población reclusa nos está señalando que aquella población que no tiene acceso a las oportunidades de la educación son las que de facto han tenido un comportamiento desviado de un proyecto de vida adecuado al marco normativo de convivencia y, por ende, al cometimiento de actos ilícitos.

${ }^{15}$ Lo cual a su vez genera desigualdades sociales de amplias proporciones: PNUD, Informe sobre Desarrollo Humano en El Salvador 2013. Imaginar un nuevo país. Diagnóstico y propuesta (San Salvador, Programa de las Naciones Unidas para el Desarrollo), 2013, 157-158 
Sin ánimo de establecer un axioma, podríamos decir que a menores posibilidades de acceso a la educación existen mayores posibilidades de desviación de una conducta racional y tendrá lugar una práctica de anti-valores. Ampliaré mi análisis proporcionando algunos datos relativos al período 20092013 en tres elementos de la situación educativa nacional: matrícula, deserción y ausentismo escolar que nos permitirá comprender el porqué nuestro país aún no tiene los niveles de escolaridad necesarios.

Un problema que históricamente ha marcado a nuestro país es que de la población en edad para ingresar a la escuela siempre hay un porcentaje significativo que queda fuera de ésta. La evolución de este dato muestra cifras ciertamente alentadoras, pero aún así persisten desafios que es necesario superar. $^{16}$

Para el caso, durante los años 2009 a 2013 la tasa neta de matrícula en educación primaria ha oscilado entre un máximo de 95\% de cobertura y un mínimo de 91\%. Esto equivale a que la matrícula en este nivel ha oscilado entre los 675,422 a 794,784 estudiantes inscritos en el sistema. En el caso del tercer ciclo de educación básica la situación es más desfavorable, ya que los porcentajes netos de matrícula han oscilado entre el 56.3\% llegando a un máximo de $67.3 \%$ lo cual significa que la matrícula oscila entre 250,924 y los 275,664 estudiantes matriculados. Finalmente, la educación media posee los niveles más bajos de matrícula: de un mínimo de 135,132 a un máximo 163,727 estudiantes inscritos en este nivel, esto es, $32.6 \%$ a $38.2 \%$ respectivamente.

Tomando únicamente el dato de aquellos niños que quedaron fuera del nivel primario de educación, tenemos que 251,503 niños se quedaron sin ingresar al sistema educativo en el período señalado. Igualmente, los estudiantes que no continuaron sus estudios en el tercer ciclo de básica fueron de $\mathbf{8 1 9 , 8 1 7}$. En el caso de la media, hay $1, \mathbf{3 6 9 , 0 8 3}$ estudiantes que no se

\footnotetext{
${ }^{16}$ Hacia finales de la década de 1960 el porcentaje de matrícula de la población edad para ingresar a la primaria era del 70\%. Tras el conflicto que atravesó el país de 1980 a 1992 esta cifra fue aumentando hasta superar a inicios del nuevo siglo el $90 \%$ de matrícula.
} 
matricularon cuando tenían la edad correspondiente para cursar ese nivel. Estos datos son preocupantes, pues la niñez y adolescencia que debería estar recibiendo algún tipo de instrucción se vuelve más vulnerable frente a diferentes problemas sociales, como lo es el de las pandillas.

En la siguiente gráfica se ilustra cómo a medida que se avanza en los niveles del sistema educativo la cantidad de estudiantes tiende a una disminución progresiva:

\section{GRÁFICA 2}

TASAS NETAS DE MATRÍCULA EN EDUCACIÓN

PRIMARIA, SECUNDARIA Y MEDIA (2009-2013)

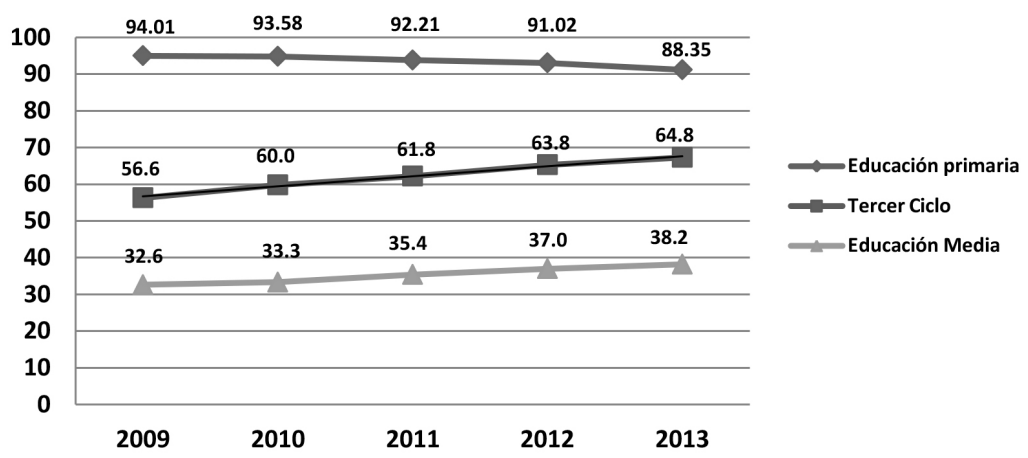

Fuente: Elaboración propia en base a: Ministerio de Educación, Tasas netas de escolarización por nivel educativo.

Para tener una idea de la cantidad de estudiantes que año con año no se matriculan en el sistema escolar, presento la siguiente gráfica de los niveles primario, secundario y de educación media: 


\section{GRÁFICA 3}

ESTUDIANTES EN EDAD ESCOLAR NO MATRICULADOS POR NIVELES EDUCATIVOS (2009-2013)

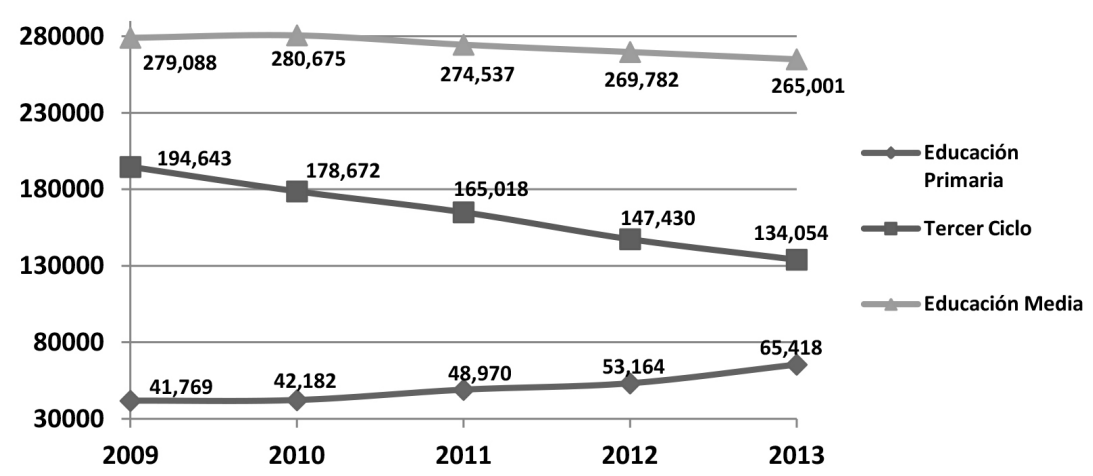

Fuente: Elaboración propia en base a: MINED,

Tasas netas de escolarización por nivel educativo.

La gráfica nos muestra población que no se matriculó en los diferentes niveles educativos a pesar que estaban en edad escolar de hacerlo. Sin embargo, existe otro grupo de población estudiantil que año con año se matriculan en la escuela pero abandonan sus estudios de manera parcial o definitiva; o sea, el sistema debe reñir con aquellos que no logran matricularse, pero también con aquellos que lo hacen pero que por diversas razones se retiran, desierta. Esto igualmente plantea ampliar el análisis referente a las tasas de matrícula, pues si bien es cierto el país ha avanzado significativamente en las últimas dos décadas en este aspecto, es preciso que el sistema logre la permanencia del estudiante en el mismo.

Para el caso, entre los años 2009 a 2013 desertaron 338,909 alumnos en el nivel de educación primaria y secundaria. En términos porcentuales ello ha representado en estos años un promedio de 5.28\% de estudiantes desertores por año. En el caso de la Educación Media, en el mismo período, desertaron 73,981 estudiantes; en términos porcentuales ha representa- 
do un promedio de $7.38 \%$ de alumnos desertores. ${ }^{17}$ Es notorio un mayor porcentaje en el caso de la Educación Media, lo cual debe representar una preocupación pues en esta es todavía menor la cantidad de estudiantes que logran acceder a este nivel con menores porcentajes de finalización de la misma. La "lógica" del sistema educativo indica, como mostré en la gráfica anterior, que cada nivel del mismo representa un filtro en el que cada vez más son menos los que van accediendo y que efectivamente logran finalizar su proceso formativo.

Debo mencionar que esta realidad no deja de ser preocupante, pues como esta tendencia se mantenga en el país será muy dificil restarle a aquellas condiciones sociales desfavorables que posibilitan el surgimiento de grupos delincuenciales como lo son las pandillas. Si bien es cierto este análisis debería desagregarse aún más en los aspectos: zona geográfica (rural y urbana), municipios con mayores niveles de deserción y posibles causas, municipios que presentan índices más bajos de matrícula en los niveles educativos, entre otros, para vislumbrar con mayor claridad la relación causal entre aquellos que optan por la vía de la delincuencia y aquellos que sí tienen oportunidades de acceso a la educación.

\section{Los Centros Educativos adscritos a los Centros de Internamiento}

La situación educativa específicamente en los Centros Escolares adscritos a los Centros de Inserción Social más un Centro Intermedio muestra que el total de personas en 2013 que se encuentra matriculadas en estos cinco CE es de 961, las cuales están distribuidas de la siguiente forma por sexo: 890 hombres, que representa el $92.61 \%$ de esta población; y 71 del sexo femenino, que representa el 7.39\%. Este dato confirma, dicho sea de paso, la tendencia a nivel global de quienes tienden a cometer hechos delictivos son

\footnotetext{
${ }^{17}$ Ministerio de Educación. Educación de El Salvador en cifras 2009-2013. Gerencia del Sistema de Estadísticas Educativas, 2014, 20
} 
mayormente hombres que las mujeres, tal y como refleja el dato global de los internos en centros penales.

La situación de matrícula en estos centros refleja una realidad similar a la de los centros penales, y es que la mayoría de personas, el 91.25\%, no ha superado los estudios de educación básica. Es importante mencionar que por las edades de las personas que pagan una pena en estos Centros deberían estar por lo menos matriculados en sexto grado de primaria, otros ubicados ya en bachillerato, pero la realidad es que existen alumnos todavía en primer grado. Solamente un 8.74\% está inscrito en el nivel de Educación Media. La siguiente gráfica ilustra la situación de la matrícula inicial para 2013 de esta población menor de edad privada de libertad.

\section{GRÁFICA 6}

POBLACIÓN INSCRITA EN EDUCACIÓN BÁSICAY MEDIA EN CENTROS DE INSERCIÓN SOCIAL

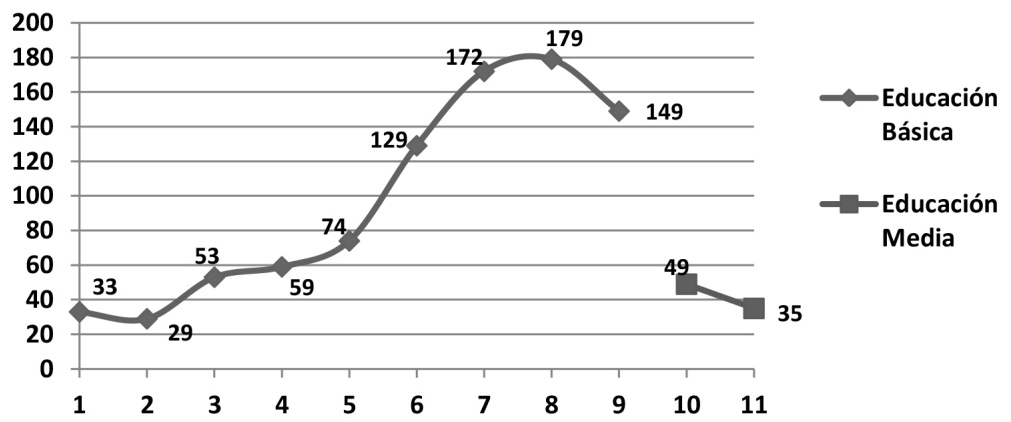

Fuente: Elaboración propia en base a MINED, Censo Escolar Inicial, 2013

A pesar de las situaciones de contingencia que se presentan en estos Centros la cifra de matrícula final, para el año que estoy analizando, arroja una cifra favorable: de la matrícula inicial de 961 únicamente 38 aparecen como desertores, mientras que de los 923 que finalizaron 837 fueron promovidos al grado siguiente y solamente 86 deberán repetir curso. 
Estas cifras nos dan una idea del movimiento de los educandos que se encuentran en los diferentes CE ubicados tanto en las CP como en los CIS. Sin embargo, debo enfatizar en el hecho de que si bien es cierto que durante el proceso de inserción social el aspecto socio educativo contribuye sustancialmente, no puede por sí solo garantizar el éxito de aquel, es decir, debe acompañarse de otros elementos como el apoyo psicológico y socio familiar. De otra parte, el solo hecho de que los internos vayan aprobando los grados educativos tampoco garantiza el éxito en el proceso de inserción social. El mejor logro que este proceso puede arrojar es el cambio de expectativa frente a la vida que nuevas oportunidades e ideas pueda abrirse en la mente de aquellos que han violado las leyes.

\section{El proceso socio educativo en los centros de inserción social: una aproximación a la problemática actual}

\section{Perfil del adolescente, situación de pandillas e infraestructura de los CIS}

Es importante señalar que cada uno de los CE ubicados en los CIS tiene características que hace que el análisis a realizar sobre estos no debe hacerse de una manera homogénea, aunque se trate de una problemática en común, pues las condiciones en que se halla la población reclusa son variadas, unas más apropiadas, otras menos. De otra parte, tampoco es viable separar el proceso estrictamente educativo con el proceso en general de inserción social cuyo principal impulsor e institución responsable es el ISNA. En otras palabras, el proceso de inserción social, en el cual va implícita la educación formal, es responsabilidad de todas las instituciones del Estado involucradas en el mismo.

La distribución geográfica de los cuatro CIS se halla concentrada principalmente en la zona occidental y paracentral: Ilopango, Tonacatepeque, Ahuachapán e Ilobasco. En la zona oriental no se cuenta aún con infraestructura para atender a esta población menor de edad. A excepción del 
CIS de Ilopango, todos albergan población masculina. La característica en común de los CIS es que el total de la población interna tiene una filiación pandilleril. Esto no siempre fue así, pues originalmente estos Centros no fueron pensados para albergar exclusivamente este tipo de población adolescente y joven con una acumulación delictiva que implica el cometimiento de homicidios. En otras palabras, el problema se ha ido agravando radicalmente, pues el delito por el que normalmente ingresan estos jóvenes es por homicidio. $^{18}$

Esta situación de pandillas implica que el tratamiento que reciban estos adolescentes no solo debe reñir con el hecho que son responsables de una falta penal, sino también con el hecho que pertenecen a estructuras delincuenciales de mayor alcance como lo son las pandillas. Por lo tanto, los ilícitos cometidos por estos adolescentes no son meros actos fortuitos, sino producto de acciones delincuenciales que sistemáticamente impulsan las pandillas en nuestro país. No se trata de adolescentes problemáticos sin más, sino de personas que conciben la estructura de la pandilla como parte vital en su modo de pensar y actuar. Por consiguiente, el tratamiento a brindar tiene que tener la capacidad de modificar radicalmente la cosmovisión de estos jóvenes.

Asimismo, el perfil de esta población menor de edad tiene como una de sus características su bajo nivel educativo, ya que la matrícula debería copar los niveles de tercer ciclo de básica y educación media, pero la realidad es que todavía hay muchos de estos jóvenes que ni siquiera han finalizado la primaria, tal y como mostré en la gráfica 6. En suma, esta población joven se caracteriza por haber cometido faltas penales como el homicidio, su bajo nivel educativo y su filiación pandilleril.

En este contexto sumamente problemático resulta difícil que en el país deban seguirse sin más los lineamientos que emanan de las normas y

\footnotetext{
18 ISNA, Una aproximación al fenómeno de las pandillas en El Salvador, Subdirección de Investigación y Estadísticas, 2011, 7. Este trabajo también indaga en las condiciones socio-familiares de origen de estos adolescentes que se incorporan a las pandillas. 18
} 
recomendaciones internacionales, entiéndase para el caso el tema de justica para menores de edad. Uno de los principios establecidos en este ámbito, y que se halla también reflejado en la Ley Penal Juvenil vigente en nuestro país, es que los tiempos, cuando se dictan medida de internamiento por parte del Juez de Menores para estos jóvenes, deben ser lapsos lo más corto posible. ${ }^{19}$ En el contexto de nuestro país y por el tipo de delito que cometen estos jóvenes, dificilmente puede seguirse al pie de la letra esta recomendación o mandato jurídico. Es más, debe considerarse un mayor tiempo de internamiento, pues eso puede dar a las autoridades responsables de la inserción social mayores posibilidades de trabajar con los jóvenes y que estos realmente experimenten un cambio en su cosmovisión y, por ende, en su conducta, pero ello es un trabajo que consiste en un proceso lento y prolongado que puede variar de acuerdo a la mentalidad específica de cada joven.

De otra parte, vale mencionar que al igual que en los CP la situación de pandillas plantea una distribución de los espacios físicos que permiten separar los diferentes bandos rivales, esto con la finalidad de evitar enfrentamientos que pueden conllevar trágicas consecuencias. En este sentido, debo mencionar que la población recluida en el CIS de Ilopango alberga población con filiación pandilleril de la M-18 como de la MS; el de Tonacatepeque, población únicamente con filiación pandilleril a la MS; el de Ahuachapán población exclusivamente con filiación a la M-18. Mientras que el CIS de Ilobasco alberga población que se ha retirado de la pandilla, entiéndase de ambas pandillas (MS y M-18). ${ }^{20}$

\footnotetext{
${ }^{19}$ En el artículo 8 de la referida Ley se encuentran establecidas, entre otras, las siguientes medidas: orientación y apoyo socio familiar; servicios a la comunidad; libertad asistida y finalmente el internamiento.

${ }^{20}$ sta separación por pandillas se realizó inicialmente en los Centros de Internamiento para Menores y luego pasó a los Centros Penales. Consultar el trabajo de El Faro titulado "El país que entregó las cárceles a sus pandilleros". Disponible en: http://www.salanegra.elfaro.net. Última consulta: 8 de enero de 2015
} 
No obstante, una norma básica de distribución de estos Centros es que posee normalmente tres "sectores". Sector es el sitio específico donde habitan los jóvenes excluyendo los espacios físicos de esparcimiento u otras actividades socio educativas, talleres de aprendizaje, religiosas, entre otras. Por ejemplo, en el caso de Ahuachapán se hallan internados jóvenes de la misma pandilla, la M-18, pero estos se hallan divididos en dos bandos: los "sureños" y los "revolucionarios", razón por la cual cada uno de estos bandos necesita convivir en un espacio separado respecto del otro; en Ilopango, esta división es lógica por la diferencia entre pandillas opuestas; en Tonacatepeque se encuentran jóvenes de la misma pandilla.

Por su lado, el "tercer sector" en estos CIS siempre se halla reservado para aquellos que por una u otra razón se encuentran enfrentados con su misma pandilla, motivo por el cual se trasladan a este sector a fin de garantizar su integridad física. En otros casos, como el de Ilobasco, este sector se considera un espacio de aislamiento que voluntariamente los jóvenes solicitan. Sin duda, estas características de los espacios físicos afectan el normal desarrollo de la atención educativa que por derecho reciben estos jóvenes, lo cual anotaré a continuación.

\section{La infraestructura de los CE, la atención educativa y actividades de formación complementarias}

Esta situación de divisionismo entre pandillas ha ido complicando el trabajo de las autoridades en relación a la atención educativa que reciben estos jóvenes. Un caso ilustrativo en este sentido es el del CE que funciona en el CIS de Ahuachapán. La infraestructura de este Centro se ha ido modificando conforme van emergiendo las diferencias entre integrantes de la misma pandilla. Como hice mención, este CIS se ha visto en la necesidad de dividirse en tres sectores. En este proceso de separación el problema que se presenta es que el espacio de aulas que funciona como CE quedó atrapado prácticamente en uno de los sectores, el número dos. De esa forma, la aten- 
ción educativa para las otras dos poblaciones se ve en mayores dificultades, pues los maestros trabajan en aulas improvisadas en pasillos al interior de los sectores, ya que es prácticamente imposible que pandilleros de bandos rivales convivan en un mismo sector.

En el caso del CIS de Ilopango se presenta también una situación dificultosa, pues a la fecha el edificio construido para el CE no es utilizado por las adolescentes privadas de libertad para recibir sus clases, en cambio, las reciben el área ubicada en los "sectores" con la limitante que son aulas muy reducidas que apenas y pueden albergar entre ocho y diez estudiantes. El edificio del CE, en cambio, es utilizado por varones menores de edad que se encuentran albergados en el CISNA y es allí donde reciben también instrucción educativa. Es importante mencionar que esta población se encuentra bajo la figura de restitución de derechos y no propiamente de inserción social, que es la que aplica a los adolescentes bajo medidas de internamiento. ${ }^{21}$

En el caso de Tonacatepeque el CE se encuentra en un espacio apropiado, ya que es una zona neutral donde pueden acceder tanto los jóvenes internos en el CIS como aquellos que pertenecen al Centro Intermedio. Lo mismo debe mencionarse del CE ubicado en el CIS de Ilobasco, pues en tanto que se encuentra un terreno neutral ambos grupos de jóvenes reciben sus clases en el CE de forma apropiada.

En un contexto como éste, la importancia de contar con un espacio neutral para el CE es vital, ya que define el área de trabajo que le permite al docente establecer un nivel básico de disciplina y rigor para un tipo de estudiante que fácilmente tiende a la indisciplina y a la desatención de las indicaciones. Esto último conlleva el hecho de que los jóvenes no reciban su proceso formativo en el CE. Las razones son diversas, por ejemplo, cuando se han generado situaciones conflictivas al interior de los CIS los maestros se han visto en la necesidad de ingresar a los "sectores" a impartir las clases a los

\footnotetext{
${ }^{21}$ La situación de este Centro es sin duda también altamente problemática y debe ser tomada en cuenta por las autoridades para brindar un tratamiento integral a estos niños y jóvenes que se hallan internos en dicho Centro.
} 
estudiantes. Que el maestro acuda a estos sectores plantea serias dificultades, pues esos espacios son asumidos por estos jóvenes como su "territorio", esto significa que ellos pueden realizar cualquier tipo de acción en estas zonas. En ocasiones las clases han tenido que ser suspendidas tras suscitarse hechos conflictivos al interior de los CIS tanto por razones de seguridad para el personal porque los jóvenes quedan en estado de tensión y retomar las clases se vuelve inconveniente. Para normalizarlos se apela a actividades deportivas hasta que se tranquilizan y nuevamente puedan retomar las clases.

Por otra parte, la situación de división en las pandillas plantea para los maestros un doble esfuerzo en la atención educativa a esta población, pues deben organizar los horarios escolares de tal modo que puedan ser atendidos los grupos por separado. Esta manera de funcionar es igual para todos los CE, pero a fin de ilustrar la situación, por ejemplo, en Ilopango, se atienden por la mañana las jóvenes pertenecientes a una pandilla, mientras que por la tarde se atiende el otro grupo pandilleril. En vista de que la educación es un derecho para esta población también aquellas personas que se encuentran en el "tercer sector" deben recibir la atención educativa, es decir, representa un esfuerzo adicional a los horarios normales de clase. En el caso de Tonacatepeque, por ejemplo, se logra atender a esta población aislada restándole una hora de las cinco que tiene la jornada escolar de la población que asiste en el turno matutino.

Es de mencionarse también que los horarios escolares deben garantizar una logística sin fallo alguno, pues estas personas de bandos contrarios no pueden siquiera cruzarse por un pasillo, ya que puede desatar una trifulca que en ocasiones ha dejado daños a la integridad fisica del personal de las instituciones, tal y como ha sucedido en algunas oportunidades en el CIS de Ilopango.

Es importante mencionar que adicionalmente al horario estrictamente escolar estos jóvenes reciben talleres de aprendizaje en oficios como una actividad permanente. Como parte del tratamiento para la inserción social se desarrollan actividades relacionadas a la sastrería, panadería, carpinte- 
ría, agricultura, computación, corte y confección, estructuras metálicas, entre otros, que prácticamente hacen que estos jóvenes permanezcan durante todo el día ocupados en alguna actividad productiva. De igual forma, en la medida que la infraestructura de cada CIS lo permite, las actividades deportivas se llevan a cabo de forma permanente. En este sentido, el CIS de Ilobasco es el que mayor espacio para este tipo de actividades tiene, cuenta además de una cancha de fútbol y baloncesto, con una de volibol.

Entretanto, el proceso educativo debe desarrollarse en estas circunstancias, algunas más favorables, otras no. A continuación señalaré las características de la educación en este contexto.

\section{Características del proceso educativo}

De acuerdo al panorama descrito anteriormente, podemos inferir que los CE que funcionan al interior de los CIS no tienen la forma de los CE tradicionales, algo que se puede comprobar desde las mismas condiciones de infraestructura y en general de las condiciones en que tiene lugar el proceso educativo en estos CE; asimismo, tampoco la labor docente debe entenderse en su sentido habitual como sucede en los contextos normales. El trabajo docente va más allá de la función de garantizar aprendizajes básicos en los estudiantes; debe hacer también las veces de una especie de guía para este tipo de jóvenes que se hallan en proceso de inserción social.

Sobre esto podemos apuntar diferentes elementos que caracterizan a todos los CE adscritos a los CIS y que pueden coadyuvar a una comprensión de la educación en estos contextos, así como algunas limitantes que se presentan a fin de ir planteando posibles soluciones.

1. La población matriculada en estos CE posee en su totalidad sobre edad en relación al grado que cursa;

2. Los grados de la primaria van cursándose bajo la modalidad acelerada, asimismo el bachillerato es cursado en modalidad a distancia; 
3. Los profesores no poseen una especialización en la atención educativa hacia este tipo de población adolescente y joven pertenecientes a pandillas;

4. No existen lineamientos específicos que orienten en el tratamiento educativo de adolescentes y jóvenes en medidas de internamiento, tomando en cuenta las características de esta población;

5. El seguimiento del proceso educativo e inserción social para esta población es fundamental para la obtención de resultados favorables (está sujeta a movilidad e incluso a recibir su formación académica bajo el acompañamiento de un tutor);

En relación a la sobreedad escolar es un hecho que de por sí representa que un maestro, sea en un contexto normal o de encierro, tendrá que orientar de un modo distinto el proceso de enseñanza aprendizaje para que el educando pueda hacerse de los conocimientos pertinentes según el nivel educativo que cursa. En el contexto de encierro es usual que los maestros de los CE realicen adecuaciones curriculares basándose en su experiencia y siguiendo un diagnóstico mínimo de las capacidades del estudiante que ingresa, ya que existe la situación que algunos de ellos que no presenta en los CE certificado oficial de aprobación del último grado que dice haber cursado.

En consonancia con la modalidad educativa ofrecida por estos CE, la cual es de aulas integradas, esta adecuación busca priorizar y establecer jerarquías en los contenidos educativos y brindar fundamentalmente al estudiante las competencias básicas, no máximas a que éstos puedan llegar, ello debido a la premura de impartir lo que normalmente debería aprenderse en dos años, hacerlo en uno solo. El maestro de este contexto de encierro piensa en el proceso que atravesará este tipo de estudiante y trata de brindarle lo más relevante de los programas educativos en un tiempo y un horario que no son de la regularidad. 
Modalidad integrada significa, valga mencionar, que los grados no van cursándose uno a uno, sino dos simultáneamente (ejemplo: segundo y tercer grado) hasta llegar al tercer ciclo de básica, el cual se cursa normalmente en estos Centros. La labor de readecuación curricular es un trabajo permanente de reformulación, de repensar el proceso de enseñanza, pues como en todo proceso educacional los grupos de estudiantes varían en capacidades e intereses. Especialmente en este contexto los CE no avanzan a ritmo uniforme, pues la situación pandilleril, esto es, los amotinamientos que puedan generarse en cada CIS, tiende irremediablemente a entorpecer el proceso formativo.

Un hecho de gran relevancia en este proceso socio educativo no es solamente la capacidad que tenga el maestro de transmitir los conocimientos a este tipo de educando, sino en general que el CE pueda brindar una oferta educativa que a esta población le motive y le resulte atractiva para que estos jóvenes asuman el proceso socio educativo como un compromiso, pensando que con la realización del mismo pueden consumar modificaciones sustanciales en sus expectativas frente a la vida. En este sentido, es importante que estos CE comporten la capacidad de ofrecer el nivel de educación básica, pero también el bachillerato y en la medida de lo posible establecer convenios con instituciones de educación superior que puedan ofrecer a estos jóvenes la posibilidad de cursar una carrera universitaria.

Sobre esto último, es ilustrativo el caso del CIS de Ilobasco, donde además de que el CE ofrece el bachillerato modalidad a distancia, también existe un convenio con una Institución de Educación Superior del sector privado que ofrece la carrera de trabajo social, donde actualmente hay más de 10 estudiantes inscritos en el cuarto ciclo. Una oferta de este tipo resulta sin lugar a dudas sumamente atractiva para esta población y puede brindarle mayores posibilidades de incorporarse a un trabajo formal al finalizar sus estudios y cumplir la medida impuesta por el Juez. 
La especialización de los docentes para laborar en este tipo de contextos resulta importante fundamentalmente por dos razones: la primera se debe a que deben tener una preparación, no solo en su ámbito disciplinar que podrían ejercer en cualquier CE del país, sino también una formación adicional para tener la capacidad de trabajar con adolescentes y jóvenes problemáticos a fin de lograr sortear las dificultades que se presentan en el proceso de enseñanza aprendizaje en estas condiciones especiales; ello implica, entre otras cosas, una inducción en la normativa jurídica relativa a las faltas cometidas por menores.Y la segunda que será funcional para retroalimentar la planta docente que trabaja en estos CE. En la mayoría de casos, los docentes que laboran en estos contextos normalmente recalan en estos CE por la necesidad de tener un empleo. En tal sentido, que exista una especialización en esta área prepara mentalmente a aquella persona hacia el tipo de contexto en que ha de laborar.

Sobre esto último es importante mencionar que los docentes que laboran en estos CE son contratados anualmente, pues la modalidad bajo la que se administran estos CE es la de los CIE. La modalidad de contratación docente es similar a la que se realizaba desde las Asociaciones Comunales de Educación (ACE) que surgieron con el modelo de Escuela EDUCO. ${ }^{22}$ Es decir, existe una presión permanente año con año sobre estos docentes en el sentido de que no gozan de estabilidad laboral. Sin embargo, es un hecho que al CIE le interesa garantizar la continuidad de estos profesores, pues ellos ya tienen un conocimiento de las leyes vinculadas a esta problemática, además de una experiencia acumulada en el trato con estos jóvenes.

Ahora bien, en el caso de que los docentes logren su estabilidad laboral mediante la contratación vía ley de salarios plantea un problema para estos CE, pues con seguridad estos solicitarían su traslado debido a que estos contextos son altamente problemáticos y cualquier maestro preferirá

\footnotetext{
${ }^{22}$ Educación con Participación de la Comunidad. Modelo educativo implementado durante la década de los noventa, principalmente orientado a la zona rural del país con el objetivo de acercar los servicios educativos a esta población tradicionalmente excluida de los mismos.
} 
trabajar en una escuela tradicional. Este dilema podría solucionarse únicamente llegando a un acuerdo con los docentes que les garantice su estabilidad laboral, pero con el compromiso de quedarse laborando en los CE. Este aspecto actualmente está trabajándose desde las instancias de coordinación del ISNA como con las del MINED.

Por otra parte, es importante que los docentes, principalmente los nuevos cuadros que vayan incorporándose al trabajo de estos $\mathrm{CE}$, tengan lineamientos básicos para el tratamiento de estos jóvenes pandilleros. Si bien el trabajo de inserción social tiene un carácter interinstitucional, en este aspecto el mayor peso de responsabilidad recae en la institución rectora de la educación, que es el MINED. No obstante, a la fecha no existe un documento de esta índole por parte de las autoridades de educación, el cual es una necesidad que debe llenarse, a juicio de los docentes que laboran en estos CE. En ese sentido, que exista la especialidad docente en áreas como esta garantiza que la planta docente de estos CE vaya retroalimentándose.

En relación a esto último, es importante aprovechar el apoyo técnico regional que brindan algunos organismos que se dedican para el caso específico de la educación en contextos de encierro. Para el caso debe recordarse que en el año 2007 se llevó a cabo en nuestro país el "Seminario nacional sobre educación en contextos de encierro", donde se contó con la participación de ponentes internacionales y se realizaron mesas de trabajo sobre el tema en diferentes áreas. Posteriormente, durante los años 20082009 se brindaron capacitaciones a los profesores que laboran en contextos de encierro, pero al parecer el esfuerzo no tuvo continuidad y no se logró aprovechar al máximo, pues un buen resultado hubiese sido la elaboración de unas normas mínimas para el tratamiento de adolescentes y jóvenes que cumplen medidas de internamiento.

El proceso educativo formal que reciben los jóvenes puede verse trastocado a causa de la movilidad a que están sujetos debido al proceso de seguimiento por la vía legal. Este proceso de seguimiento de conducta y readaptación de estos jóvenes se da por medio de los informes individuales 
que son enviados al juez de menores por parte de las autoridades del CIS. Según el criterio de este último un joven puede recibir la medida de libertad asistida, la cual consiste en "otorgar la libertad al menor, obligándose éste a cumplir programas educativos, a recibir la orientación y el seguimiento del los tribunales". ${ }^{23}$ Lo que esto implica es abandonar el recinto del CIS y por consiguiente la asistencia al CE.

Sin duda, ello provoca que el estudiante abandone la escuela, al menos parcialmente, pues los profesores continúan trabajando con los jóvenes, pero bajo otra metodología, la cual consiste en seguir las orientaciones de un tutor. Los jóvenes se presentan cada cierto tiempo al CE para entregar trabajos y realizar evaluaciones, con esta medida no pierden la realización de su proceso socioeducativo y garantiza el compromiso de estos con la inserción social. En el caso de la población masculina que recibe medidas de internamiento más prolongadas la movilidad es menor. Por otra parte, la población femenina recibe lapsos más breves de internamiento, por lo que en este caso se dificulta aún más el proceso socio educativo de las internas.

Esta medida de seguimiento es fundamental no solo en lo referente al proceso socio educativo, sino en general al proceso de inserción social, pues poco contribuiría el hecho de que estos jóvenes reciban su libertad asistida o en forma permanente, sino se trabaja en modificar el contexto social en el que viven. En otras palabras, el asunto se vuelve más complejo, pues el trabajo de inserción debe involucrar en forma decisiva a la familia del joven para que éste no se torne un reincidente. Sin embargo, ello representa un problema, pues normalmente estos jóvenes provienen de familias desintegradas en el mejor de los casos, pues es notable que muchos de ellos no tienen una familia pues durante el cumplimiento de las medidas de internamiento ni siquiera reciben visita.

\footnotetext{
${ }^{23}$ Ley Penal Juvenil, artículo 14
} 


\section{Conclusiones y recomendaciones}

La complejidad del asunto tratado nos lleva a realizar la interrogante: ¿pueden estos jóvenes realmente insertarse a la sociedad y ser funcionales a esta? No cabe duda que muchos de ellos efectivamente modifican su conducta y expectativas ante la vida. Sin embargo, la misma pertenencia a pandillas hace que éstos desarrollen una especie de código de lealtad hacia estas estructuras que vuelve realmente difícil una incorporación normal a la sociedad. Por otra parte, ello también puede conllevarles trágicas consecuencias, pues muchos jóvenes que abandonan la pandilla son asesinados por los que otrora fueran sus compañeros.

Esto último nos plantea señalar la siguiente conclusión, y es que no es viable la inserción social cuando aquellos jóvenes que recuperan su libertad retornan a las mismas condiciones sociales que dieron origen a que estas personas se desviaran del marco social. Dicho en otras palabras, el proceso de inserción social debe tomar en cuenta las condiciones relacionadas al proceso que tiene lugar en los CIS, pero también las condiciones externas, principalmente socio-familiares, para que estos jóvenes no se tornen reincidentes, pues de esa manera se tira al traste todo el esfuerzo de las autoridades por insertar a estos jóvenes. Esta situación vuelve realmente dificil una normal readaptación a la vida social de esta población y constituye el principal enemigo de las instituciones que impulsan tal proceso.

De otra parte, el esfuerzo de este trabajo nos permite visualizar el perfil de esta población. Su elaboración responde al hecho de que ello puede posibilitar una comprensión del problema y de resolverlo en el largo plazo desde sus orígenes, pues si solo se trabaja sobre las consecuencias dejando de lado las causas no cabe duda que el problema social continuará reproduciéndose. Ello plantea la necesidad no solo de trabajar la mentalidad del joven, sino modificar el contexto del cual este procede para que puedan tener efectos duraderos el trabajo de inserción social. 
Algunos de los aspectos sobre los cuales debe reflexionarse y que tienen que ver estrictamente con los procesos al interior de los CIS son los siguientes:

La no convivencia entre diferentes grupos pandilleriles. Esta imposibilidad de que estas personas con diferentes filiaciones de pandillas no puedan relacionarse normalmente plantea una situación complicada, pues lo que refleja es el nivel de deshumanización al que estas personas han llegado bajo una cosmovisión absolutamente errada. En lo mediato las autoridades deberán trabajar en la mentalidad de ellos para superar estas barreras que les impiden relacionarse normalmente con otras personas.

Las condiciones de infraestructura de estos Centros, mencionada más arriba y que es semejante a la de los CP, fomenta ese compañerismo entre miembros de pandilla que prácticamente vuelve dificil el trabajo individual con cada uno de estos jóvenes para ir eliminando todos los prejuicios e ideas erróneas que tienen. Lo que esto significa es que no existen celdas donde transiten de manera individual o en pareja, sino que todos están en un mismo espacio físico. La noción de estos Centros como medida de castigo también es importante para que aquellos tengan claro que deben modifica su conducta para que no tengan que atravesar nuevamente ese proceso.

En relación a algunos aspectos relacionados al proceso educativo debo mencionar los siguientes aspectos:

Existe una cantidad limitada de docentes que laboran en estos CE, lo cual genera una sobrecarga laboral que impide un tratamiento más específico y, en consecuencia, de mayor calidad del maestro hacia el educando. De igual manera, se debe mejorar las condiciones laborales de este sector, ya que es una labor altamente compleja y que ciertamente muy pocos maestros quieren realizar. La oferta de laborar en estos contextos debería resultar atractiva para atraer personal que labore en estos CE.

La infraestructura de los CE debe ajustarse de tal modo que todos los internos puedan acceder al CE, esto debido a los fraccionamientos en sectores propios de estos recintos y que tienen como principal causa la división pandilleril. 
Es necesaria la especialización docente en el área de atención educativa para esta población adolescente y joven sujetos de responsabilidad penal, cuya principal característica es la filiación a pandillas. Ello permitirá desarrollar adecuadamente un trabajo tanto en la atención educativa como en el proceso general de inserción social. De otra parte, permitirá ir retroalimentando la planta docente que labora en estos contextos.

Deben ampliarse la oferta en aquellos CIS que aún no cuentan con modalidad de educación media e igualmente deben ampliarse las alianzas con instituciones de educación superior que permitan el acceso a los internos a una carrera profesional. Esto sin duda tendrá mayor impacto para evitar la reincidencia de los jóvenes y permitirá un abandono definitivo de su antigua vida.

En general, el concepto de inserción social que las autoridades impulsan refleja el máximo esfuerzo que las autoridades puedan realizar, en términos de la inversión de recursos humanos y materiales que ello implica. La idea desplegada en estos CIS es correcta, sin embargo, por las mismas condiciones de los jóvenes y de igual forma las condiciones externas, pueden afectar sustancialmente la labor de inserción social impulsada por las autoridades.

Finalmente, no me cabe la menor duda que el modelo implementando desde los CIS debería ser emulado por las autoridades que dirigen los Centros Penitenciarios, ya que de esa forma se evitaría el ocio carcelario e involucraría activamente a todos los presidiarios en actividades productivas que les motiven a readaptarse a la vida civil. 
1

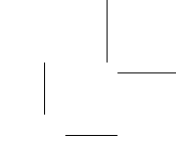




\section{REFERENCIAS BIBLIOGRÁFICAS}

\section{Libros y artículos}

Amaya, Luis Enrique y Martínez, Juan José. “Sureños en El Salvador: un acercamiento antropológico a las pandillas de deportados" en Realidad $y$ Reflexión, Enero-Junio, N 39, 2014

El Salvador. Corte Suprema de Justicia. Ley Penal Juvenil. San Salvador: Sección de Publicaciones de la Corte Suprema de Justicia, 2004

Instituto de Medicina legal, "Total de homicidios año 2013, según base de datos del Instituto de Medicina Legal, cotejada y consensuada con F.G.R. y la P.N.C."

ISNA. Programa de atención en la medida administrativa de resguardo, sin sello editorial

ISNA. Programa de atención en las medidas en medio abierto, sin sello editorial

ISNA. Programa de atención en la medida de internamiento, sin sello editorial

ISNA, Una aproximación al fenómeno de las pandillas en El Salvador. San Salvador: Subdirección de Investigación y Estadísticas, 2011

ISNA. Programa marco para la atención integral de adolescentes sujetos a responsabilidad penal juvenil. San Salvador, Mayo de 2013

Ministerio de Educación. Educación de El Salvador en cifras 2009-2013. San Salvador: Gerencia del Sistema de Estadísticas Educativas, 2014

PNUD, Informe sobre Desarrollo Humano en El Salvador 2013. Imaginar un nuevo país. Diagnóstico y propuesta (San Salvador, Programa de las Naciones Unidas para el Desarrollo), 2013 


\section{Fuentes en soporte electrónico}

http://www.gobiernoabierto.gob.sv/

"El país que entregó las cárceles a sus pandilleros". Disponible en: http:// www.salanegra.elfaro.net.

http://www.mined.gob.sv/index.php/estadisticas-educativas 